\title{
Humanidades Médicas e seu Lugar no Currículo: Opiniões dos Participantes do Cobem/2017
}

\section{Medical Humanities and their Place in the Curriculum: Opinions of Cobem/2017 Participants}

\author{
Jaqueline Santos Barboza ${ }^{\mathrm{I}, \mathrm{II}}(\mathbb{D}$ \\ Helena Maria dos Santos Felício ${ }^{\text {II }}$ (D)
}

\section{PALAVRAS-CHAVE}

- Currículo.

- Educação Médica.

- Humanidades.

- Ciências Humanas.

- Abordagem Interdisciplinar do Conhecimento na Educação.
${ }^{1}$ Universidade José do Rosário Vellano, Alfenas, Minas Gerais, Brasil.

${ }^{\mathrm{II}}$ Universidade Federal de Alfenas, Alfenas, Minas Gerais, Brasil. 


\section{KEYWORDS}

- Curriculum.

- Medical Education.

- Humanities.

- Human Sciences.

- Interdisciplinary Approach to Knowledge in Education.

Recebido em 21/9/19

Aceito em 26/11/19

\section{INTRODUÇÃO}

As humanidades médicas referem-se ao conjunto de disciplinas cujos objetivos educacionais e conteúdos trazem para o campo teórico e prático da Medicina contribuições da Filosofia, Ética, Psicologia, Antropologia, Artes, Sociologia, História, Política, Educação, ou seja, disciplinas que buscam fundamentos nas Ciências Humanas e Sociais para compreender a condição humana no âmbito da Medicina e desenvolver competências para o cuidar ${ }^{1}$.

As Ciências Humanas e Sociais sempre abordaram questões relativas à saúde ou à doença. Quando essas disciplinas aportaram na educação médica, introduziram a discussão sobre a prática de relações comunicativas entre médico e paciente discutidas nos âmbitos educacional e ético e, mais recentemente, no campo da bioética clínica e em pesquisa ${ }^{2}$. Assim, na Medicina, comprometeram-se com conjuntos bem definidos de problemas sobre a condição humana (morte, morrer, confidencialidade, aborto, personalidade, alocação de fundos a serviços de saúde etc.) e o uso de estratégias interdisciplinares para abordar esses problemas ancorados na práxis das profissões de saúde ${ }^{1}$. Para Rios ${ }^{2}$, as humanidades médicas e as Ciências Humanas e Sociais em saúde abordam temas humanísticos, porém com diferentes ênfases: as humanidades médicas estão mais voltadas às relações humanas no âmbito da intersubjetividade, e as Ciências Humanas e Sociais em saúde, às relações no âmbito da sociedade.

As disciplinas que compõem o rol das humanidades médicas propiciam maior compreensão das expressões do sofrimento humano e das manifestações socioculturais dos adoecimentos, assim como de habilidades de comunicação e construção de vínculos que possibilitam a interação necessária a qualquer ato médico.

\section{ABSTRACT}

There is a growing perception that medical training with an exclusive focus on biological disciplines is insufficient for the understanding of the complexity and individuality of human illness. We start from the assumption that medical humanities make an important contribution to medical education, through references to expanded understanding of the health-disease process and to the organization of health care, that is, directed at humanization. This work sought to assess the perceptions of a group of students, teachers and coordinators of a medical course on the integration of the disciplines of medical humanities in the curricula of medical courses. The descriptive, quantitative and cross-sectional research consisted of the application of a structured questionnaire to a group of participants of the $55^{\text {th }}$ Brazilian Congress of Medical Education (COBEM, Porto Alegre, RS, October/2017), considered to be a convenience sample. The event participants were approached during the intervals of the conferences and invited to answer a questionnaire that sought opinions on the insertion and the integration of the disciplines of the human sciences into the curricula of the medical courses. institution (private, public or confessional) and the role of the respondent (teacher, student or coordinator). A total of 234 (two hundred and thirty-four) individuals answered the questionnaires, which were submitted to descriptive statistical analysis. The analysis showed that the medical humanities are perceived as important for the medical formation, but there is no consensus about their being crucial for the acquisition of humanistic skills. There are interdisciplinary curricular experiences; however, not all of them perceive the subjects of humanities at different times in the medical curriculum or articulated with other disciplines. While interdisciplinary experiences are perceived more easily, the cross-sectional articulation of humanistic topics appears to a lesser degree. According to the answers, the disciplines remain concentrated only in the initial periods; however, the perception of sufficiency discloses quite divided opinions. There is also a slight difference in opinion about the articulation of the humanities in the basic and advanced cycles. The lower perception of integration of the humanities appears in practices that integrate teaching-service-community. 


\section{PERCURSO METODOLÓGICO}

Trata-se de uma pesquisa descritiva, com abordagem quantitativa e de corte transversal, na qual se utilizou um questionário estruturado aplicado aos participantes do $55^{\circ}$ Congresso Brasileiro de Educação Médica (Cobem, Porto Alegre, RS, outubro de 2017), considerada assim uma amostra de conveniência. Essa técnica de amostragem não probabilística consiste em selecionar uma amostra representativa de uma população-alvo e que esteja acessível. Nesse caso, os participantes do Cobem (docentes, discentes e coordenadores de curso médico) representam um grupo de profissionais envolvidos com a educação médica e, por estarem agregados no mesmo espaço físico em um evento de porte nacional que discute justamente essa temática, foram considerados uma amostragem representativa e disponível.

Os participantes do evento foram abordados nos intervalos das conferências e convidados a responder um questionário contendo 14 perguntas, em escala Likert, que buscavam opiniões sobre a inserção e a integração das disciplinas das Ciências Humanas nos currículos dos cursos de Medicina. O questionário continha ainda a caracterização da amostra quanto à região de origem, ao âmbito jurídico da instituição (privada, pública ou confessional) e ao papel do respondente (docente, discente ou coordenador/a). Antes de sua aplicação, o questionário foi submetido a préteste com discentes de curso de Medicina e docentes da área médica.

Tabularam-se os dados obtidos no modo simplificado, considerando as entradas "discordo", "concordo", "não concordo nem discordo" e "em branco" (para as questões não respondidas). Os dados foram tabulados no software de criação e edição de planilhas Microsoft Excel e analisados por meio de análise estatística descritiva, com auxílio de tabelas de frequências, em que se apresentam as contagens em cada categoria (frequência absoluta) e os percentuais que essas contagens representam no total (frequência relativa).

\section{Considerações éticas}

De acordo com a Resolução do Conselho Nacional de Saúde e Ministério da Saúde (CNS/MS) no 510/2016 ${ }^{5}$, as pesquisas que utilizam dados obtidos por meio de coleta de opinião com participantes não identificados (que corresponde à nossa pesquisa) não necessitam de registro ou avaliação pelo Comitê de Ética em Pesquisa e Comissão Nacional de Ética em Pesquisa (CEP/Conep).

\section{RESULTADOS E DISCUSSÃO}

Obtiveram-se 234 questionários válidos, dos quais 163 eram de estudantes (70\%), 56 de docentes (24\%) e 15 de coordenadores $(6 \%)$ de cursos de Medicina. Em relação à instituição dos entrevistados, 58\% (132 respondentes) estudam ou trabalham em universidade ou faculdades particulares, e 42\% (94 respondentes), em instituição pública. Além disso, a maioria dos participantes é da Região Sul (41\%). A Região Centro-Oeste é a que contém menos entrevistados em análise (3\%).

A análise dos resultados concentrou-se, por conveniência, nos papéis desempenhados pelos respondentes, e, assim, os dados são apresentados e discutidos considerando as respostas dos docentes, discentes e coordenadores.

O questionário buscou primeiro verificar o conhecimento da matriz curricular e das disciplinas de humanidades médicas das escolas dos respondentes. Dos entrevistados, a grande maioria diz conhecer a matriz curricular do curso de Medicina da sua escola. Em relação ao cargo, os coordenadores apresentam a maior porcentagem de conhecimento (93\%), seguidos dos professores (89\%) e, por fim, dos estudantes (83\%).

Em relação ao conhecimento das humanidades médicas, também a maioria dos respondentes (76\%) afirmou conhecer tais disciplinas nos currículos. Os coordenadores foram os que apresentaram maior porcentagem de conhecimento das disciplinas de humanidades médicas do curso de Medicina (93\%).

De modo geral, os respondentes da pesquisa afirmaram que as humanidades médicas são fundamentais para a formação do médico, correspondendo a mais de $90 \%$ dos respondentes, como mostra a Tabela 1.

É evidente uma unanimidade sobre a importância das disciplinas de humanidades no currículo. Entretanto, questionados sobre a relação entre essas disciplinas e a aprendizagem de habilidades humanísticas, apenas a metade dos respondentes consideram que há dependência entre elas, como mostra a Tabela 2.

A porcentagem de entrevistados que discordam da afirmação é relativamente alta, sendo $33 \%$ dos coordenadores, $38 \%$ dos professores e $29 \%$ dos estudantes. Nesse sentido, parece que os respondentes atribuem a aprendizagem dessas habilidades a elementos da formação médica que extrapolam o currículo formal, como convívio e identificação com docentes e preceptores.

Sobre esse aspecto não formal do currículo, é preciso considerar o caráter oculto e não previsível das muitas determinações no complexo sistema curricular, especialmente as que se relacionam ao ambiente e que contribuem na socialização dos discentes via normas, valores e sistemas de comunicação que regulam o acesso do estudante ao conhecimento, em um contexto de relações sociais características ${ }^{6}$. Como ressalta Alonso ${ }^{6}$, existe um campo de contradição entre o que se expressa no currículo e sua

\begin{tabular}{|c|c|c|c|c|c|c|c|c|c|c|}
\hline \multicolumn{11}{|c|}{ Percepção da relevância das humanidades na formação médica } \\
\hline \multicolumn{11}{|c|}{ Questão 3: As disciplinas das humanidades médicas são fundamentais para a formação do médico. } \\
\hline & \multicolumn{2}{|c|}{ Concordo } & \multicolumn{2}{|c|}{ Discordo } & \multicolumn{2}{|c|}{$\begin{array}{l}\text { Não concordo } \\
\text { nem discordo }\end{array}$} & \multicolumn{2}{|c|}{ Em branco } & \multicolumn{2}{|c|}{ Total } \\
\hline & Freq. & $\%$ & Freq. & $\%$ & Freq. & $\%$ & Freq. & $\%$ & Freq. & $\%$ \\
\hline Coordenador(a) & 14 & $93 \%$ & 0 & $0 \%$ & 0 & $0 \%$ & 1 & $7 \%$ & 15 & $100 \%$ \\
\hline Estudante & 150 & $92 \%$ & 2 & $1 \%$ & 11 & $7 \%$ & 0 & $0 \%$ & 163 & $100 \%$ \\
\hline Professor(a) & 51 & $91 \%$ & 0 & $0 \%$ & 1 & $2 \%$ & 4 & $7 \%$ & 56 & $100 \%$ \\
\hline
\end{tabular}

Fonte: Elaborada pelas autoras.

$3 \mid$\begin{tabular}{l|l} 
REVISTA BRASILEIRA DE EDUCAÇÃO MÉDICA \\
\hline 44 (1) : e028; 2020
\end{tabular} 
vivência na prática, o que nos alerta para a necessidade de conscientizar os professores, por meio da reflexão crítica, dos mecanismos ocultos que contribuem para a desvirtuação e perversão do projeto inicial de formação e de socialização contidos no currículo.

Nesse sentido, Rios ${ }^{4}$ argumenta que, embora o aprendizado de valores e atitudes se dê pela observação dos mestres em ação no dia a dia, é fundamental que nos currículos médicos existam disciplinas específicas da área e temas humanísticos transversais, em diferentes momentos e durante toda a graduação.

Além disso, a contribuição das Ciências Humanas e Sociais nas discussões éticas é fundamental para a formação do estudante (como a segurança do paciente e a diversidade na garantia de direitos sociais, questões de gênero e etnia, entre outras condições) e uma das definições das Diretrizes Curriculares Nacionais dos Cursos de Graduação em Medicina, que preveem ainda uma estrutura curricular tendo as Ciências Humanas e Sociais como eixo transversal na formação profissional ${ }^{7}$.
A transversalidade curricular refere-se a uma integração temática que culmina na presença de um núcleo temático em todas as disciplinas. Segundo Menezes e Santos ${ }^{8}$, enquanto a interdisciplinaridade questiona a visão compartimentada da realidade sobre a qual a escola se constituiu, a transversalidade diz respeito à compreensão dos diferentes objetos de conhecimento, possibilitando a referência a sistemas construídos na realidade dos alunos que promovem uma compreensão alargada dos diferentes objetos de conhecimento.

Sobre experiências curriculares interdisciplinares e transversais, $80 \%$ dos coordenadores, $66 \%$ dos professores e $59 \%$ dos estudantes respondentes da pesquisa de opinião concordaram que suas escolas oferecem ensino a partir dessa estratégia.

Nenhum coordenador respondente discorda da afirmação de que a instituição oferece experiências interdisciplinares. A maior discordância aparece entre os discentes, evidenciando possível descompasso entre a percepção dos discentes e dos coordenadores sobre as atividades

\begin{tabular}{|c|c|c|c|c|c|c|c|c|c|c|}
\hline \multicolumn{11}{|c|}{ Tabela 2} \\
\hline \multicolumn{11}{|c|}{ Questão 5: A aprendizagem das habilidades humanísticas depende das disciplinas de humanidades. } \\
\hline & \multicolumn{2}{|c|}{ Concordo } & \multicolumn{2}{|c|}{ Discordo } & \multicolumn{2}{|c|}{$\begin{array}{l}\text { Não concordo } \\
\text { nem discordo }\end{array}$} & \multicolumn{2}{|c|}{ NR } & \multicolumn{2}{|c|}{ Total } \\
\hline & Freq. & $\%$ & Freq. & $\%$ & Freq. & $\%$ & Freq. & $\%$ & Freq. & $\%$ \\
\hline Coordenador(a) & 7 & $47 \%$ & 5 & $33 \%$ & 3 & $20 \%$ & 0 & $0 \%$ & 15 & $100 \%$ \\
\hline Estudante & 80 & $49 \%$ & 48 & $29 \%$ & 34 & $21 \%$ & 1 & $1 \%$ & 163 & $100 \%$ \\
\hline Professor(a) & 30 & $54 \%$ & 21 & $38 \%$ & 3 & $5 \%$ & 2 & $4 \%$ & 56 & $100 \%$ \\
\hline
\end{tabular}

Fonte: Elaborada pelas autoras.

\begin{tabular}{|c|c|c|c|c|c|c|c|c|c|c|}
\hline \multicolumn{11}{|c|}{$\begin{array}{c}\text { Tabela } 3 \\
\text { Percepção das experiências interdisciplinares }\end{array}$} \\
\hline \multicolumn{11}{|c|}{ Questão 6: O currículo médico da minha escola possibilita experiências interdisciplinares. } \\
\hline & \multicolumn{2}{|c|}{ Concordo } & \multicolumn{2}{|c|}{ Discordo } & \multicolumn{2}{|c|}{$\begin{array}{l}\text { Não concordo } \\
\text { nem discordo }\end{array}$} & \multicolumn{2}{|c|}{ Em branco } & \multicolumn{2}{|c|}{ Total } \\
\hline & Freq. & $\%$ & Freq. & $\%$ & Freq. & $\%$ & Freq. & $\%$ & Freq. & $\%$ \\
\hline Coordenador(a) & 12 & $80 \%$ & 0 & $0 \%$ & 2 & $13 \%$ & 1 & $7 \%$ & 15 & $100 \%$ \\
\hline Estudante & 96 & $59 \%$ & 36 & $22 \%$ & 29 & $18 \%$ & 2 & $1 \%$ & 163 & $100 \%$ \\
\hline Professor(a) & 37 & $66 \%$ & 8 & $14 \%$ & 7 & $13 \%$ & 4 & $7 \%$ & 56 & $100 \%$ \\
\hline
\end{tabular}

Fonte: Elaborada pelas autoras.

Tabela 4

Percepção da longitudinalidade das humanidades

Questão 7: As temáticas de humanidades aparecem em diversos momentos no currículo médico, inclusive dentro de outras disciplinas.

\begin{tabular}{|c|c|c|c|c|c|c|c|c|c|c|}
\hline & \multicolumn{2}{|c|}{ Concordo } & \multicolumn{2}{|c|}{ Discordo } & \multicolumn{2}{|c|}{$\begin{array}{l}\text { Não concordo } \\
\text { nem discordo }\end{array}$} & \multicolumn{2}{|c|}{ Em branco } & \multicolumn{2}{|c|}{ Total } \\
\hline & Freq. & $\%$ & Freq. & $\%$ & Freq. & $\%$ & Freq. & $\%$ & Freq. & $\%$ \\
\hline Coordenador(a) & 14 & $93 \%$ & 0 & $0 \%$ & 1 & $7 \%$ & 0 & $0 \%$ & 15 & $100 \%$ \\
\hline Estudante & 104 & $64 \%$ & 36 & $22 \%$ & 23 & $14 \%$ & 0 & $0 \%$ & 163 & $100 \%$ \\
\hline Professor(a) & 36 & $64 \%$ & 11 & $20 \%$ & 8 & $14 \%$ & 1 & $2 \%$ & 56 & $100 \%$ \\
\hline
\end{tabular}

Fonte: Elaborada pelas autoras. 


\begin{tabular}{|c|c|c|c|c|c|c|c|c|c|c|}
\hline \multicolumn{11}{|c|}{ Tabela 5} \\
\hline \multicolumn{11}{|c|}{ Questão 8: As disciplinas de humanidades articulam-se com outras disciplinas e campos de conhecimento, no currículo de Medicina da minha escola. } \\
\hline & \multicolumn{2}{|c|}{ Concordo } & \multicolumn{2}{|c|}{ Discordo } & \multicolumn{2}{|c|}{$\begin{array}{l}\text { Não concordo } \\
\text { nem discordo }\end{array}$} & \multicolumn{2}{|c|}{ Em branco } & \multicolumn{2}{|c|}{ Total } \\
\hline & Freq. & $\%$ & Freq. & $\%$ & Freq. & $\%$ & Freq. & $\%$ & Freq. & $\%$ \\
\hline Coordenador(a) & 11 & $73 \%$ & 2 & $13 \%$ & 1 & $7 \%$ & 1 & $7 \%$ & 15 & $100 \%$ \\
\hline Estudante & 82 & $50 \%$ & 44 & $27 \%$ & 35 & $21 \%$ & 2 & $1 \%$ & 163 & $100 \%$ \\
\hline Professor(a) & 30 & $54 \%$ & 9 & $16 \%$ & 12 & $21 \%$ & 5 & $9 \%$ & 56 & $100 \%$ \\
\hline
\end{tabular}

Fonte: Elaborada pelas autoras.

interdisciplinares. De maneira semelhante, $93 \%$ dos coordenadores, $64 \%$ dos professores e $64 \%$ dos estudantes concordaram com a afirmativa de que as temáticas de humanidades aparecem em diversos momentos no currículo médico, inclusive dentro de outras disciplinas.

A articulação das temáticas humanísticas, entretanto, é percebida em menor medida, como mostra a Tabela 5 .

Enquanto as experiências interdisciplinares são percebidas com maior facilidade, a articulação interdisciplinar e transversal das temáticas humanísticas não aparece com expressão na opinião dos respondentes.

Segundo Fazenda ${ }^{9}$, a interdisciplinaridade consiste na interação entre duas ou mais disciplinas, que pode ir da simples comunicação de ideias à integração mútua dos conceitos epistemológicos, metodológicos, dos procedimentos, dos dados e da organização referentes ao ensino e à pesquisa. Um grupo interdisciplinar compõe-se de pessoas que receberam sua formação em diferentes domínios do conhecimento (disciplinas) com seus métodos, conceitos, dados e termos próprios, mas que trabalham de forma coordenada. A autora aponta ainda que deve haver uma relação de reciprocidade, de mutualidade ou de copropriedade que possibilita o diálogo. Nesse sentido, depende basicamente de uma atitude, na qual a colaboração entre diversas disciplinas conduz a uma "interação" - uma intersubjetividade.

De fato, a inserção das humanidades médicas, como disciplinas estanques nos currículos, desarticuladas dos outros conteúdos e descontextualizadas da prática médica, expõe aos alunos informação humanística, mas não garante necessariamente formação humanística, como aponta Pereira ${ }^{1}$. É preciso repensar as configurações curriculares, de modo que favoreçam minimamente a interdisciplinaridade $e$ transversalidade, para uma integração realmente efetiva dessas temáticas como formativas na educação médica.

O fato de cada disciplina confinar seus saberes e compartimentalizar seus conteúdos impossibilita, muitas vezes, o exercício da conexão entre os conhecimentos por parte do profissional de saúde em formação, e isso impacta o perfil do profissional, que se constrói naturalmente a partir de um paradigma reducionista e fragmentado. Implica, certamente, a conformação da sua visão do mundo e as suas práticas de atenção à saúde das pessoas ${ }^{10}$.

Ao assumirem concepções rasas sobre essa face de articulação, as instituições correm o risco de não possibilitarem uma compreensão integral do ser humano e do processo saúde-doença, que passa necessariamente por uma abordagem interdisciplinar e transversal, como destacado por Feuerwerker ${ }^{11}$, mantendo fragmentadas as discussões e apropriações conceituais.

Outro aspecto de fragmentação aparece em relação ao agrupamento das disciplinas de humanidades no currículo, que, na opinião dos respondentes, concentram-se no primeiro ano (segundo semestre) e no segundo ano (quarto semestre).

Sabemos que a experienciação das discussões humanísticas no início do curso médico pode promover a aproximação dos graduandos aos seus conceitos e às suas reflexões, na medida em que já se sabe que a escolha pelo curso médico costuma ser justificada pelo desafio intelectual e pela preocupação com questões humanitárias, como "ajudar pessoas", "fazer o bem" e manter uma "boa relação médico-paciente", conforme nos mostra Scheffer ${ }^{12}$, ainda que as humanidades não se limitem a essas concepções.

Entretanto, se essas disciplinas permanecem concentradas apenas nos períodos iniciais, provocam ruptura nas discussões. Isoladas nos anos iniciais, carecem de diálogo com as demais disciplinas da graduação e ficam condicionadas à condição de corpo estranho no ensino médico. Resulta disso a visão de sua pouca serventia, distante do que é tido como o escopo central da profissão médica e assim "dispensáveis" ou desmotivadoras ${ }^{4}$. Essa condição se agrava na transição entre os ciclos básico e avançado, quando costuma ocorrer um abrupto afastamento das disciplinas básicas em função das práticas clínica e hospitalar.

Sobre a divisão curricular em ciclos tradicionais (básico e avançado ou profissional), $53 \%$ dos coordenadores, $63 \%$ dos professores e $72 \%$ dos estudantes afirmam que o currículo possui tal divisão. Dos entrevistados, os coordenadores são os que mais discordam da afirmação (47\%). Entretanto, apenas $47 \%$ dos coordenadores, $39 \%$ dos professores e $31 \%$ dos

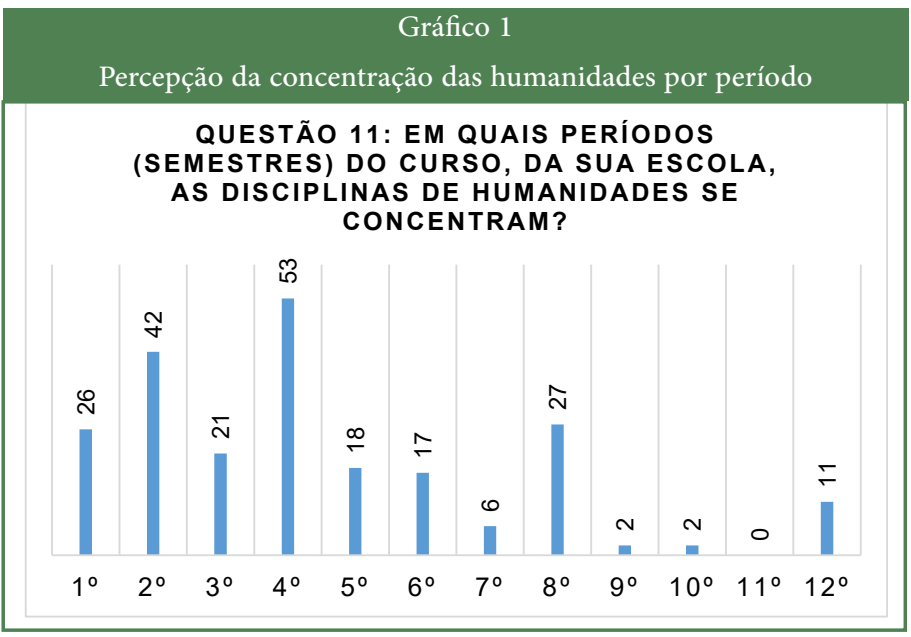

Fonte: Elaborado pelas autoras. 
estudantes afirmam que as disciplinas de humanidades aparecem em todos os ciclos do curso. Dentre os entrevistados, os estudantes são os que mais discordam da afirmação (49\%). Apenas os coordenadores apresentaram porcentagem de concordância maior que a porcentagem de discordância.

Não é incomum observar que, de fato, as humanidades ocupam espaços confinados de saber nos currículos, como mostram as respostas. Comumente restritas ao ciclo básico, as humanidades não se apresentam em níveis crescentes de complexidade, ao contrário, observa-se um decréscimo quantitativo em sua inserção ao longo dos ciclos.

Esse fenômeno quase sempre está associado à atribuição de diferentes valores entre as disciplinas:

Em geral, as disciplinas que lidam com os aspectos biológicos e com as intervenções no corpo, mediadas por procedimentos, são espaços que recebem maior investimento por parte da comunidade acadêmica. Pouca atenção está voltada àquelas que propõem reflexões e ações no campo da ética, das humanidades, do ambiente, das relações interpessoais, intrapsíquicas e das interações sociais ${ }^{10}$ (p. 264).

Trata-se de um cenário que se configura a partir de um ordenamento disciplinar característico do racionalismo científico. $O$ perfil do profissional tende, assim, a se construir naturalmente a partir de um paradigma reducionista e fragmentado, o que certamente impacta sua visão do mundo e suas práticas de atenção à saúde das pessoas ${ }^{10}$.

A relação da instituição com os sujeitos aprendentes está permeada pela "transmissão" de determinados conhecimentos curriculares, e, consequentemente, essa escolha é feita excluindo-se outros. Apple ${ }^{13}$ analisa esse movimento pensando nas relações entre o conhecimento aberto e o encoberto que se ensina nas escolas, bem como os princípios de seleção e organização desse conhecimento. Na medida em que entendemos a cultura e o conhecimento como produtos das relações sociais e, portanto, das relações hierárquicas, assimétricas e de poder, o currículo (como o conhecimento e a cultura) não pode ser considerado fora dessas relações, pois é a oficialização dos saberes contidos e excluídos da formação. Assim, é possível que a escola médica, tanto quanto outras instituições, se porte como agente de uma hegemonia cultural e ideológica, reproduzindo certas desigualdades.

Ainda assim, uma discreta maioria dos coordenadores (53\%) e dos estudantes (56\%) considera suficiente a carga horária das humanidades no currículo médico na sua escola. Já pequena maioria dos professores (52\%) considera a carga horária insuficiente.

Considerando que não há uma equilibrada distribuição de carga horária entre os diferentes campos disciplinares ao longo da graduação, como mostra o Gráfico 1, é possível que as percepções de suficiência ou insuficiência sejam afetadas negativamente por essa disposição quantitativa desequilibrada, ainda que não se possa fazer uma correspondência direta entre essa percepção e a carga horária dos currículos analisados. Para responder a essa questão, a análise documental dos currículos a que se referem os respondentes seria fundamental.

Contudo, sabemos que a simples inserção das humanidades médicas como disciplinas estanques e isoladas contribui para a descontextualização da reflexão que lhe é característica, podendo gerar problemas motivacionais, de percepção da aplicabilidade e relevância do que se aprende. Além disso, tende a uma visão simplista, compartimentada e estática da realidade, ao insuficiente diálogo com o meio, ao pouco estabelecimento de nexos entre os conteúdos, que acabam por ser vistos como "gavetas que se abrem e fecham sobre si mesmos" (p. 406) ${ }^{14}$.

Atualmente, as Diretrizes Curriculares Nacionais do Curso de Graduação em Medicina recomendam que a estrutura dos cursos médicos

\begin{tabular}{|c|c|c|c|c|c|c|c|c|c|c|}
\hline \multicolumn{11}{|c|}{ Tabela 6} \\
\hline \multicolumn{11}{|c|}{ Questão 10: No currículo da minha escola, as disciplinas de humanidades aparecem em todos os ciclos do curso. } \\
\hline & \multicolumn{2}{|c|}{ Concordo } & \multicolumn{2}{|c|}{ Discordo } & \multicolumn{2}{|c|}{$\begin{array}{l}\text { Não concordo } \\
\text { nem discordo }\end{array}$} & \multicolumn{2}{|c|}{ Em branco } & \multicolumn{2}{|c|}{ Total } \\
\hline & Freq. & $\%$ & Freq. & $\%$ & Freq. & $\%$ & Freq. & $\%$ & Freq. & $\%$ \\
\hline Coordenador(a) & 7 & $47 \%$ & 3 & $20 \%$ & 4 & $27 \%$ & 1 & $7 \%$ & 15 & $100 \%$ \\
\hline Estudante & 51 & $31 \%$ & 80 & $49 \%$ & 31 & $19 \%$ & 1 & $1 \%$ & 163 & $100 \%$ \\
\hline Professor(a) & 22 & $39 \%$ & 24 & $43 \%$ & 7 & $13 \%$ & 3 & $5 \%$ & 56 & $100 \%$ \\
\hline
\end{tabular}

Fonte: Elaborada pelas autoras.

\begin{tabular}{|c|c|c|c|c|c|c|c|c|}
\hline \multicolumn{9}{|c|}{ Tabela 7} \\
\hline \multicolumn{9}{|c|}{ Questão 12: A carga horária das humanidades no currículo médico da sua escola é: } \\
\hline & \multicolumn{2}{|c|}{ Suficiente } & \multicolumn{2}{|c|}{ Insuficiente } & \multicolumn{2}{|c|}{ Em branco } & \multicolumn{2}{|c|}{ Total } \\
\hline & Freq. & $\%$ & Freq. & $\%$ & Freq. & $\%$ & Freq. & $\%$ \\
\hline Coordenador(a) & 8 & $53 \%$ & 7 & $47 \%$ & 0 & $0 \%$ & 15 & $100 \%$ \\
\hline Estudante & 92 & $56 \%$ & 67 & $41 \%$ & 4 & $2 \%$ & 163 & $100 \%$ \\
\hline Professor(a) & 25 & $45 \%$ & 29 & $52 \%$ & 2 & $4 \%$ & 56 & $100 \%$ \\
\hline
\end{tabular}

Fonte: Elaborada pelas autoras.

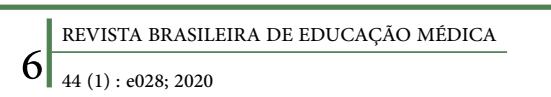


tenha como eixo do desenvolvimento curricular as necessidades de saúde dos indivíduos e das populações identificadas pelo setor saúde. As escolas médicas devem ainda promover a integração e a interdisciplinaridade em coerência com esse eixo de desenvolvimento curricular, buscando integrar as dimensões biológicas, psicológicas, étnico-raciais, socioeconômicas, culturais, ambientais e educacionais. Além disso, devem inserir o aluno nas redes de serviços de saúde, consideradas como espaço de aprendizagem, desde as séries iniciais e ao longo do curso de graduação de Medicina, a partir do conceito ampliado de saúde, considerando que todos os cenários que produzem saúde são ambientes relevantes de aprendizagem? ${ }^{7}$.

De acordo com as percepções dos respondentes, a maioria dos coordenadores (67\%) considera que os conteúdos das humanidades médicas, em sua escola, não estão articulados às práticas na comunidade. Já em relação aos professores, $45 \%$ consideram que os conteúdos não estão articulados às práticas na comunidade, enquanto $27 \%$ consideram que os conteúdos estão pouco vinculados. Além disso, $25 \%$ não souberam responder. Dos estudantes entrevistados, $46 \%$ consideram que os conteúdos não estão articulados às práticas na comunidade, $26 \%$ consideram que os conteúdos estão pouco vinculados e $23 \%$ não souberam responder, como mostra a Tabela 8 .

Uma baixa interação das discussões das humanidades na integração ensino-serviço-comunidade, por exemplo, indica pouca articulação teórico-prática entre a concepção do cenário de ensino e suas proposições conceituais, como integralidade do cuidado e humanização das ações em saúde. Os princípios e as diretrizes do Sistema Único de Saúde (SUS) e os conceitos das humanidades devem, assim, ser alinhados às proposições que intentam cuidado integral, centrado na pessoa, na família e na comunidade, pautado na concepção de clínica ampliada e compartilhada.

Abordar a integração curricular das humanidades médicas implica, portanto, reconhecer o desafio que se apresenta para a educação médica do século XXI, na medida em que superar o paradigma do ensino centrado na doença, no professor e na linearidade dos conteúdos requer a construção de novos olhares sobre a teoria e prática curriculares, o que desde já se configura como renovação.

\section{CONSIDERAÇÕES FINAIS}

A importância da adequada integração das humanidades nos currículos médicos é um dado consolidado na literatura referente à formação médica, na medida em que promove a elucidação de questões profundamente humanas, bem como a compreensão dos processos interativos, relacionais e comunicacionais na prática médica, abordando o homem em sua complexidade e na multidimensionalidade do viver-adoecer-morrer. Por sua potencialidade filosófica e reflexiva, as humanidades contribuem para o autoconhecimento do sujeito que aprende, auxiliando na tomada de decisões em uma perspectiva clínica ampliada e compartilhada. Além disso, favorecem a construção de vínculos efetivos com a população a quem se destinam os serviços de saúde, de forma social e culturalmente contextualizada.

Quando se reflete sobre os currículos médicos e as humanidades, faz-se pertinente pensar como as diferentes propostas curriculares contribuem para a aquisição desse repertório ampliado de capacidades e atitudes diversificadas e transversais. Mas não se restringe a isso. É preciso verificar como essas temáticas têm impactado os sujeitos desse processo.

Em relação aos limites do estudo, sabe-se que uma amostra formada por procedimentos de seleção não aleatórios não garante a representatividade da população-alvo - a amostra de 234 questionários válidos, nesse caso, é uma pequena quantidade de respondentes, dificultando uma avaliação de maior expressividade no contexto nacional. Tal limitação, contudo, não impede inferências gerais sobre a situação analisada.

Ademais, a pesquisa evidenciou que as humanidades médicas são percebidas como importantes na formação, mas não há consenso sobre serem fundamentais na aquisição de habilidades humanísticas. As experiências interdisciplinares curriculares existem, porém nem todos percebem as temáticas de humanidades em diversos momentos no currículo médico ou articuladas a outras disciplinas. Enquanto as experiências interdisciplinares são percebidas com maior facilidade, a articulação transversal das temáticas humanísticas aparece em menor medida. De acordo com as respostas, as disciplinas permanecem concentradas apenas nos períodos iniciais, contudo a percepção de suficiência apresenta opiniões divididas. Também há discreta diferença na opinião sobre a articulação das humanidades nos ciclos básico e avançado. A menor percepção de integração das humanidades aparece nas práticas que integram ensino-serviço-comunidade.

É possível que o que consideramos como pouco do ponto de vista teórico seja percebido como suficiente na prática formativa, dadas as concepções hegemônicas que atravessam as formatações curriculares há décadas. Mas também é possível que a cultura e o movimento de intensas discussões dos elementos psicossociais na contemporaneidade estejam, de fato, universalizando percepções. $O$ movimento de padronizações diagnósticas e a massiva medicalização de processos psicossociais têm trazido à tona a demanda de discussão das humanidades anterior e exterior ao contexto universitário. Daí a importância da integração das humanidades na formação universitária - trazer à teorização os elementos experienciados na vida, não negligenciando ou massificando os sujeitos, mas impulsionando em sua formação aquilo que ele se propõe a ser.

\begin{tabular}{|c|c|c|c|c|c|c|c|c|c|c|}
\hline \multicolumn{11}{|c|}{ Tabela 8} \\
\hline \multicolumn{11}{|c|}{$\begin{array}{l}\text { Percepção das humanidades na interação ensino-serviço-comunidade } \\
\text { nteúdos das humanidades médicas, em sua escola, estão articulados às práticas na comunidade? }\end{array}$} \\
\hline & \multicolumn{2}{|c|}{ Sim } & \multicolumn{2}{|c|}{ Pouco } & \multicolumn{2}{|c|}{ Não } & \multicolumn{2}{|c|}{ Não sei } & \multicolumn{2}{|c|}{ Total } \\
\hline & Freq. & $\%$ & Freq. & $\%$ & Freq. & $\%$ & Freq. & $\%$ & Freq. & $\%$ \\
\hline Coordenador(a) & 1 & $7 \%$ & 3 & $20 \%$ & 10 & $67 \%$ & 1 & $7 \%$ & 15 & $100 \%$ \\
\hline Estudante & 7 & $4 \%$ & 43 & $26 \%$ & 75 & $46 \%$ & 38 & $23 \%$ & 163 & $100 \%$ \\
\hline Professor(a) & 2 & $4 \%$ & 15 & $27 \%$ & 25 & $45 \%$ & 14 & $25 \%$ & 56 & $100 \%$ \\
\hline
\end{tabular}

Fonte: Elaborada pelas autoras.

\begin{tabular}{l|l}
7 & REVISTA BRASILEIRA DE EDUCAÇÃO MÉDICA \\
\hline 44 (1) : e028; 2020
\end{tabular} 


\section{REFERÊNCIAS}

1. Pereira RTMC. O ensino da medicina através das humanidades médicas: análise do filme And the band played on e seu uso em atividades de ensino/aprendizagem em educação médica. São Paulo. Tese [Doutorado em Doenças Infecciosas e Parasitárias] Universidade de São Paulo; 2005.

2. Rios IC. Humanidades médicas como campo de conhecimento em Medicina. Rev. bras. educ. méd. 2016;40(1):21-9.

3. Ayres JRCM, Rios IC, Schraiber LB, Falcão MTC, Mota A. Humanidades como disciplina da graduação em Medicina. Rev. bras. educ. méd. 2013;37(3):455-63.

4. Rios IC. Subjetividade contemporânea na educação médica: a formação humanística em Medicina. São Paulo. Tese [Doutorado em Medicina Preventiva] - Universidade de São Paulo; 2010

5. Brasil. Ministério da Saúde. Conselho Nacional de Educação. Resolução $\mathrm{n}^{\circ} 510$, de 7 de abril de 2016. Dispõe sobre as normas aplicáveis a pesquisas em Ciências Humanas e Sociais cujos procedimentos metodológicos envolvam a utilização de dados diretamente obtidos com os participantes ou de informações identificáveis ou que possam acarretar riscos maiores do que os existentes na vida cotidiana. Diário Oficial da União 24 maio 2016; Seção 1, p. 44.

6. Alonso MLG. Inovação curricular, formação de professores e melhoria da escola: uma abordagem reflexiva e reconstrutiva sobre a prática da inovação/formação. Braga. Tese [Doutorado em Estudos da Criança] - Universidade do Minho; 1998.

7. Brasil. Ministério da Educação e Cultura. Conselho Nacional de Educação. Câmara de Educação Superior. Resolução no 3, de 20 de junho de 2014. Institui Diretrizes Curriculares Nacionais do Curso de Graduação em Medicina e dá outras providências. Diário Oficial da União 23 jun 2014; Seção 1, p. 8.

8. Menezes ET, Santos TH. Transversalidade (verbete). Dicionário Interativo da Educação Brasileira - Educabrasil. São Paulo: Midiamix; 2001 [acesso em 12 maio 2018]. Disponível em: http://www. educabrasil.com.br/transversalidade/.

9. Fazenda ICA. Integração e interdisciplinaridade no ensino brasileiro: efetividade ou ideologia. 6. ed. São Paulo: Edições Loyola; 2011.
10. Albuquerque VS, Batista RS, Tanji S, Moco ETM. Currículos disciplinares na área de saúde: ensaio sobre saber e poder. Interface (Botucatu) 2009;13(31):261-72.

11. Feuerwerker LM. Além do discurso de mudança na educação médica: processos e resultados. Rio de Janeiro: Hucitec; 2002.

12. Scheffer M, coordenador. Demografia médica no Brasil 2018. São Paulo: Departamento de Medicina Preventiva da Faculdade de Medicina da USP, Conselho Regional de Medicina do Estado de São Paulo, Conselho Federal de Medicina; 2018.

13. Apple MW. Ideología y currículo. Traducción Rafael Lassaletta. Madri: Akal; 2008.

14. Alonso MLG. Para uma teoria compreensiva sobre integração curricular: o contributo do Projeto "PROCUR". Infância e educação: investigação e práticas 2002;5: 62-88.

\section{CONTRIBUIÇÃO DOS AUTORES}

Msc. Jaqueline Santos Barboza é autora principal, responsável pela concepção, desenho do estudo, coleta, análise e interpretação dos dados e organização do manuscrito. Dra. Helena Maria dos Santos Felício é orientadora da pesquisa, responsável pelos aportes teóricos e revisão crítica do conteúdo. Ambas as autoras participaram da redação do artigo.

\section{CONFLITO DE INTERESSES}

As autoras declaram não possuir qualquer tipo de interesse de natureza pessoal, comercial, política, financeira ou outros que configurem Conflito de Interesses.

\section{ENDEREÇO PARA CORRESPONDÊNCIA}

Jaqueline Santos Barboza.

Rua Gabriel Moura Leite, 704, vila Formosa. Alfenas-MG.

CEP 37131-444.

E-mail: jaquelinesb_psi@hotmail.com 